\title{
Resolutividade da atenção básica em saúde bucal em municípios do estado da Paraíba, Brasil
}

\author{
Resolvability of primary care in oral health in the State of Paraíba, \\ Brazil
}

Carmellyo Pires Leite Santiago (https://orcid.org/0000-0001-8863-6596) ${ }^{1}$

Denise de Fátima Barros Cavalcante (https://orcid.org/0000-0002-9166-0367) ${ }^{2}$

Glaucia Maria Bovi Ambrosano (https://orcid.org/0000-0001-5950-6370) ${ }^{2}$

Antônio Carlos Pereira (https://orcid.org/0000-0003-1703-8171) ${ }^{2}$

Edson Hilan Gomes de Lucena (https://orcid.org/0000-0003-3431-115X) ${ }^{1}$

Yuri Wanderley Cavalcanti (https://orcid.org/0000-0002-3570-9904) ${ }^{1}$

Wilton Wilney Nascimento Padilha (https://orcid.org/0000-0003-3056-9145) ${ }^{1}$
${ }^{1}$ Programa de Pós-

Graduação em Odontologia.

Departamento de Clínica de Odontologia Social. Centro de Ciências da Saúde. Universidade Federal da Paraíba. Campos I, Cidade Universitária. 58051-900.

João Pessoa PB Brasil. wiltonpadilha@

yahoo.com.br

${ }^{2}$ Departamento de

Odontologia Social.

Faculdade de Odontologia de Piracicaba. Universidade Estadual de Campinas. Piracicaba SP Brasil.

\begin{abstract}
Oral health resolvability in primary care of municipalities in the State of Paraíba, Brazil, was analyzed, and a cross-sectional study was conducted, using an inductive approach, comparative procedure and indirect documentation. The Resolvability Indicator (RI) consisted of the ratio between the number of Completed Treatments and First Programmatic Dental Consultations in municipalities in Paraíba $(n=223)$, between 2011 and 2014, by using data collected from the DATASUS/TABNET platform. The following explanatory variables were considered: Coverage of First Programmatic Dental Consultation (CFPDC), Coverage of Primary Care Teams (CPCT), Coverage of Oral Health Teams (COHT), Coverage of Family Health Teams (CFHT), Percentage of Tooth Extraction (PTE), Gross Domestic Product (GDP), Municipal Human Development Index (MHDI) and Gini Coefficient (GC). Descriptive statistics and negative binomial multiple regression were performed $(\alpha=0.05)$. The median RI in 2011, 2012, 2013 and 2014 was, respectively, 20.4, 17.5, 15.2 and 15.7. There was a positive association between $R I$ and CFPDC $(P R=1.14, C I=$ 1.02-1.28), CPCT (PR =1.02, CI =1.01-1.03), in addition to a negative association. between RI and year $(P R=0.83$; $I C=0.74-0.94)$. The resolvability of oral health in primary care is influenced by coverage-related factors.
\end{abstract}

Key words Oral health, Health services research, Primary health care
Resumo Analisou-se a resolutividade da atenção básica em saúde bucal nos municípios do estado da Paraíba, Brasil. Trata-se de um estudo transversal de abordagem indutiva, com procedimento comparativo e técnica de documentação indireta. $O$ indicador de resolutividade (IR) constituiu da razão entre o número de tratamentos concluídos e primeiras consultas odontológicas programáticas dos municípios paraibanos $(n=223)$ entre 2011 e 2014, utilizando dados coletados da plataforma Datasus/ TABNET. Foram consideradas variáveis explicativas: cobertura de primeira consulta odontológica programática (CPCOP), cobertura de equipes de atenção básica (CEAB), cobertura de equipes de saúde bucal (CESB), cobertura de equipes de saúde da família (CESF), percentual de exodontia (PE), Produto Interno Bruto (PIB), Indice de Desenvolvimento Humano Municipal (IDHM) e coeficiente de Gini (CG). Os dados foram analisados por estatística descritiva e regressão múltipla binomial negativa $(\alpha=0,05)$. As medianas do IR em 2011, 2012, 2013 e 2014 foram, respectivamente, 20,4, 17,5, 15,2 e 15,7. Houve associação positiva do IR com CPCOP $(R P=1,14$; $I C=$ 1,02-1,28), CEAB ( $R P=1,02 ; I C=1,01-1,03), e$ negativa com ano $(R P=0,83 ; I C=0,74-0,94)$. $A$ resolutividade em saúde bucal na atenção básica é influenciada por fatores relacionados à cobertura. Palavras-chave Saúde bucal, Pesquisa sobre serviços de saúde, Atenção primária à saúde 


\section{Introdução}

A atenção básica em saúde corresponde ao contato preferencial do usuário com o Sistema Único de Saúde (SUS) no Brasil. Apresenta função de promoção, proteção e recuperação da saúde e deve possibilitar acesso contínuo, de qualidade e resolutivo, de modo a estimular melhoria dos indicadores de saúde da população ${ }^{1}$.

O Ministério da Saúde formalizou e regulamentou a criação das equipes de saúde bucal nos serviços de atenção básica no ano 2000. Em 2003, lançou a Política Nacional de Saúde Bucal - Programa Brasil Sorridente, que buscou ampliar o acesso da população aos cuidados odontológicos e elevar a resolutividade no âmbito da saúde bucal. Assim, são necessárias investigações sobre o impacto dessas políticas ${ }^{2}$.

É crescente o esforço para institucionalizar a avaliação em saúde no Brasil e no mundo ${ }^{3-5}$. Essas práticas visam promover direcionamento no planejamento e gestão da rede assistencial de saúde, contribuindo para reordenar ações e serviços ${ }^{2,6,7}$. O uso de parâmetros de avaliação no monitoramento sistemático da saúde bucal na atenção básica ainda é incipiente e pontual. Atualmente, segue em curso uma estratégia de amplitude nacional que pretende estimular e fortalecer os processos de avaliação e monitoramento. O desempenho de equipes de saúde bucal tem sido acompanhado por ciclos de avaliação sistemática do Programa Nacional de Melhoria do Acesso e da Qualidade (PMAQ) na atenção básica ${ }^{8}$. Entretanto, a avaliação sistemática do PMAQ não é suficiente para análise do processo de implantação e ampliação da oferta da atenção em saúde bucal na atenção básica. Além disso, as medidas avaliativas do PMAQ se concentram sobre intervenções de mudança do processo de trabalho no presente, que visam ampliar o acesso e qualidade do serviço no futuro. É estratégico, portanto, que a avaliação do desempenho de equipes de saúde bucal possa ser retrospectiva e prospectiva, a partir do uso de sistemas de informação, de maneira que o gestor e a sociedade sejam capazes de acompanhar o desempenho da oferta dos serviços do SUS.

A resolutividade dos serviços de saúde consiste na capacidade de ofertar cuidados que atendam as demandas da população, de casos simples até os mais complexos, desde a consulta inicial em nível básico até a solução do problema, com possível alcance em níveis mais elevados de atenção'. O entendimento a respeito da resolutividade da assistência é importante para a reorganiza- ção da qualidade do serviço ofertado ${ }^{10}$. Embora sejam observados avanços recentes nas práticas de promoção da saúde bucal, o debate sobre resolutividade permanece vivo nos meios político, científico e de gestão de serviços, devido à necessidade de se verificar o impacto desses avanços ${ }^{11}$.

Unidades de atenção básica que funcionam adequadamente, de forma resolutiva, oportuna e humanizada, deveriam ser capazes de resolver cerca de $85 \%$ dos problemas de saúde da população e direcionar o percentual restante para unidades de maiores níveis de complexidade ${ }^{12}$. No entanto, a resolutividade dos serviços de saúde no Brasil é um tema ainda pouco abordado na literatura, de modo que não existem modelos sólidos validados para a avaliação da resolutividade em saúde bucal na atenção básica. Dessa forma, pesquisadores, gestores e sociedade não possuem parâmetros de análise definidos para identificação do quão resolutivo é o serviço. Portanto, justifica-se a necessidade de ampliar análises e propostas de monitoramento da resolutividade da atenção básica em saúde bucal, contribuindo para maior conciliação entre resultados de pesquisa e práticas profissionais efetivas ${ }^{13}$.

Indicadores de saúde são usados para monitorar, avaliar e subsidiar decisões dos profissionais de saúde sobre serviços ofertados, bem como verificar a evolução na população ao longo do tempo no território. Essas características são traçadas no Manual Instrutivo PMAQ para a razão entre tratamento concluído e primeira consulta odontológica programática, indicador que avalia a relação entre acesso da população aos serviços de saúde e a resolução dos planos de tratamento traçados. Cada indicador demanda qualidade de critérios, como elaboração e precisão dos dados, validade, confiabilidade, sensibilidade, especificidade, mensurabilidade, relevância e custo-efetividade $^{14-16}$.

Segundo o último Censo, realizado em 2010, a Paraíba possui tamanho populacional estimado em 3.766.528 habitantes, distribuídos em 223 municípios, com cobertura de equipes de atenção básica em 94,4\% deles para o mesmo ano e com proporção de cobertura populacional estimada por equipes de saúde da família, entre 2011 e 2014, acima de $91 \%{ }^{17}$. A análise da relação entre indicadores da atenção básica em saúde bucal e condições socioeconômicas e demográficas municipais contribuem para o entendimento das desigualdades em saúde, sobretudo no acesso e na utilização dos serviços ${ }^{2,18}$.

Relações de diferentes fatores com a resolutividade da atenção básica em saúde bucal são 
apresentadas em estrutura conceitual na Figura 1. Indicadores de coberturas de equipe de atenção básica (CEAB) e de equipe de saúde bucal (CESB) dão uma perspectiva da abrangência da rede assistencial de saúde, no sentido de atingir seus potenciais beneficiários. A cobertura de primeira consulta odontológica programática (CPCOP) se refere ao indicador que tem por objetivo analisar o acesso da população à rede. $\mathrm{O}$ percentual de exodontias (PE) em relação aos demais procedimentos básicos tem potencial de sugerir melhoras no tratamento ofertado. Os dados socioeconômicos de Produto Interno Bruto (PIB), coeficiente de Gini (CG) e Índice Desenvolvimento Humano Municipal (IDHM) poderiam interferir na autonomia e nas decisões da população de um local. Fatores subjetivos, como satisfação do usuário com o serviço ofertado ${ }^{19}$ e sua percepção em relação à habilidade do cirurgião dentista e ao atendimento acolhedor da equipe de saúde, podem ser decisivos na adesão ao tratamento proposto. Esses fatores podem impactar direta ou indiretamente no desfecho avaliado, "indicador de resolutividade". Dessa forma, este estudo analisou a resolutividade da atenção básica em saúde bucal e quais fatores impactaram seus resultados nos municípios paraibanos entre os anos 2011 e 2014 .

\section{Métodos}

Trata-se de um estudo transversal de abordagem indutiva, com procedimento comparativo e documentação indireta. Analisou-se a resolutividade da atenção básica em saúde bucal e fatores associados nos municípios $(\mathrm{n}=223)$ do estado da Paraíba, Brasil, no período de 2011 a 2014.

Foram coletados dados de produção dos 223 municípios, porém houve a exclusão de $99 \mathrm{mu}$ nicípios cujos dados registrados na base do Sistema de Informações da Atenção Básica (SIAB) não possibilitaram completar o cálculo do indicador para os quatro anos cobertos pelo estudo, de modo que a amostra foi composta por 124 municípios.

Foram utilizados dados consolidados referentes aos tipos de atendimento do cirurgião dentista, coletados no SIAB. Valores de primeira consulta odontológica programática (PCOP) - referentes a exames clínicos odontológicos realizados com a finalidade de diagnóstico e, necessariamente, elaboração de um plano preventivo-terapêutico (PPT) - e de tratamento concluído (TC) - momento em que se finaliza o PPT - compuseram o cálculo da variável de- pendente indicador de resolutividade ${ }^{20}$ para cada município, pela equação:

$$
I R=\frac{T C}{P C O P} \times 100
$$

Indicadores relacionados à cobertura da assistência à saúde (cobertura de primeira consulta odontológica programática - CPCOP, cobertura de equipes de atenção básica - CEAB, cobertura de equipes de saúde bucal - CESB, proporção de cobertura de equipes de saúde da família - CESF e percentual de exodontias - PE) e à condição socioeconômica dos municípios (PIB, IDHM e CG) compuseram as variáveis independentes (Figura 1).

Para obter o valor da CPCOP, utilizou-se a razão entre número de primeiras consultas odontológicas programáticas e a estimativa da população coberta por equipes de atenção básica em junho do respectivo ano multiplicada por 100. A escolha do mês junho se deu por estar em posição intermediária no ano, pela praticidade e por semelhança com o monitoramento por três meses centrais utilizado pelo $\mathrm{PMAQ}^{8}$ e foi utilizada somente para esse indicador. Destaca-se ainda que a variável de cobertura, em geral, não sofreu variação significativa ao longo do ano. A escolha de um mês intermediário do ano evitou que os dados sofressem possível influência de período eleitoral. Repetiram-se os valores do PIB de 2013 para análise do ano de 2014, em virtude de não terem sido divulgados os valores deste ano no momento da coleta dos dados. O IDHM e o CG foram repetidos em toda série temporal, por se tratarem de dados informados no último Censo realizado no Brasil, em 2010.

As análises foram realizadas no programa Statistical Analysis System (SAS, University Edition, SAS Campus Drive, Cary, NC), considerando nível de significância de $5 \%$ bicaudal. O indicador de resolutividade se ajustou à distribuição binomial negativa, sendo realizada uma análise de regressão múltipla binomial negativa.

Inicialmente, todas as variáveis independentes compuseram o modelo de regressão múltipla binomial negativa. Para ajuste do modelo, realizou-se a remoção das variáveis com p-valor $>0.20$. Os ajustes dos modelos foram avaliados pelos deviance/grau de liberdade e corrected Akaike information criterion (AICc), calculados pelo procedimento PROC GENMOD do programa estatístico. A partir dos coeficientes do modelo de regressão binomial negativa, foram estimadas as magnitudes de efeito pelas razões de prevalência (RP) e p-valores (Pr > ChiSq). 


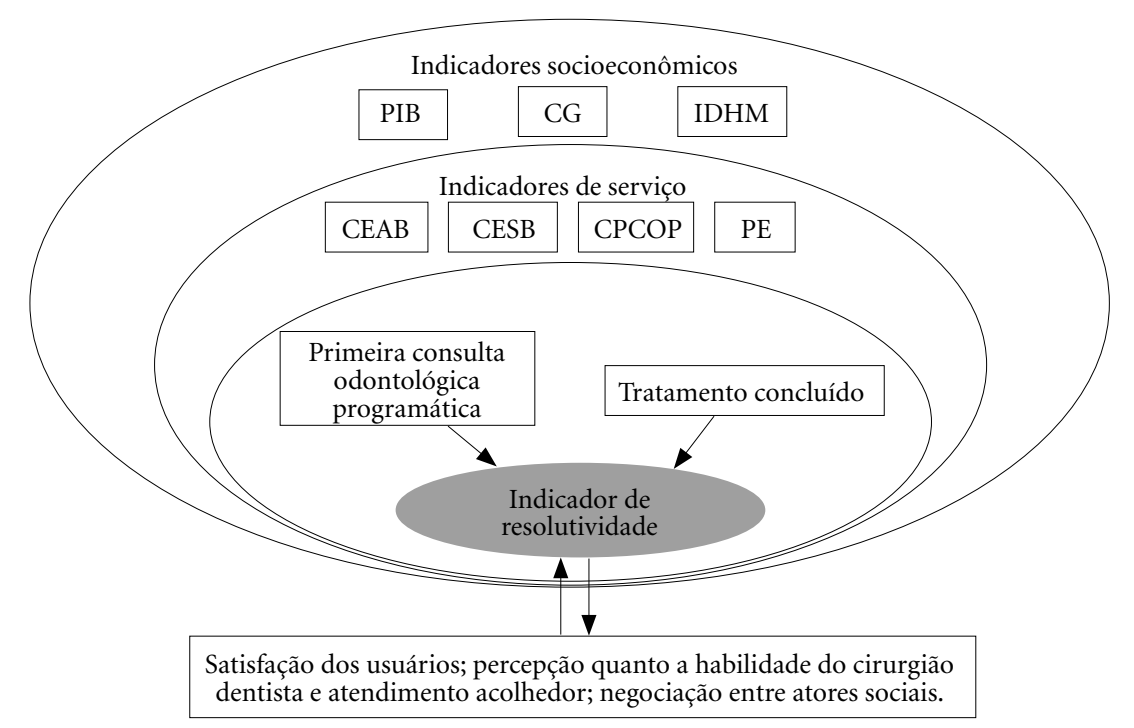

Figura 1. Hierarquização de fatores que impactam os resultados do indicador de resolutividade.

Fonte: Elaborada pelos autores.

\section{Resultados}

O número de municípios que possibilitaram calcular o IR em toda série temporal foi de 124 $(55,6 \%)$, com tamanhos populacionais que variaram entre 1.752 e 780.738 habitantes. Os municípios que não permitiram calcular o IR apresentaram tamanho populacional entre 1.243 e 105.531 pessoas. A Tabela 1 apresenta o indicador de resolutividade e dados relacionados a procedimentos básicos e à cobertura assistencial. Os dados de IR não apresentaram distribuição normal, portanto foram representados pelas medianas. A Tabela 2 apresenta a descrição dos dados sociodemográficos municipais.

A Tabela 3 apresenta resultados de associações estatisticamente significantes para o indicador de resolutividade. Apenas as variáveis ano, cobertura de primeira consulta odontológica programática, cobertura de equipes de atenção básica e PIB compuseram o modelo de regressão múltipla binomial negativa. Após ajuste do modelo, não foi constatada associação do IR com percentual de exodontias, cobertura de equipes de saúde bucal, cobertura de equipes de saúde da família, IDHM e CG ( $\mathrm{p}>0,05)$.

\section{Discussão}

Para um país das dimensões do Brasil, com 5.570 municípios, população estimada em mais de 208 milhões de pessoas ${ }^{21}$ e um sistema nacional de saúde que cobre cerca de $75 \%$ da população na atenção básica ${ }^{22}$, monitorar resolutividade de ações de saúde na atenção básica é um grande desafio. Portanto, uma avaliação de indicadores de cobertura e produção por meio de sistemas de informação pode viabilizar a avaliação sistemática de gestores, usuários e profissionais inseridos no SUS. Dessa forma, espera-se que a vigilância em saúde direcione o serviço para sua qualificação continuada.

O IR investigado neste estudo pretende avançar as avaliações de cobertura e de produção para o acompanhamento e monitoramento da resolutividade, entendida como uma forma de identificar a efetividade das ações de saúde da rede assistencial ${ }^{23}$. Nesse sentido, buscam-se dados que possam apoiar as análises sobre a questão.

O cálculo da razão entre TC e PCOP passou a ser utilizada em 2015, no terceiro ciclo do PMAQ, para montar estratégias para qualificar, acompanhar e avaliar o processo de trabalho das equipes de saúde. Numa abordagem lógica, o resultado da razão deveria variar entre 0 e 1 , uma vez que, em princípio, não se pode concluir tratamentos não iniciados. Valores muito abaixo 
Tabela 1. Valores máximos e mínimos, mediana e distribuição por quartis das variáveis indicador de resolutividade (IR) ${ }^{\mathrm{a}}$, primeira consulta odontológica programática (PCOP) ${ }^{\mathrm{b}}$, tratamento concluído $(\mathrm{TC})^{\mathrm{b}}$, percentual de exodontias relacionadas aos demais procedimentos odontológicos básicos $(\mathrm{PE})^{\mathrm{a}}$, cobertura de primeira consulta odontológica programática $(\mathrm{CPCOP})^{\mathrm{a}}$, cobertura de equipes de atenção básica $(\mathrm{CEAB})^{\mathrm{a}}$, cobertura de equipes de saúde bucal $(\mathrm{CESB})^{\mathrm{a}}$ e cobertura de equipes de saúde da família $(\mathrm{CESF})^{\mathrm{a}}$ no período de 2011 a 2014 para a Paraíba $(\mathrm{n}=124 ;$ municípios/quartil = 31).

\begin{tabular}{|c|c|c|c|c|c|c|}
\hline \multirow[t]{2}{*}{ Variável } & \multirow[t]{2}{*}{ Ano } & \multirow[t]{2}{*}{ Mínimo } & $\begin{array}{c}\text { Primeiro } \\
\text { quartil }\end{array}$ & Mediana & $\begin{array}{c}\text { Terceiro } \\
\text { quartil }\end{array}$ & \multirow[t]{2}{*}{ Máximo } \\
\hline & & & $25 \%$ & $50 \%$ & $75 \%$ & \\
\hline \multirow[t]{4}{*}{$\mathrm{IR}^{\mathrm{a}}$} & 2011 & 1,1 & 10,4 & 20,4 & 41,2 & $560,0^{*}$ \\
\hline & 2012 & 1,0 & 10,9 & 17,5 & 30,0 & $518,4^{*}$ \\
\hline & 2013 & 0,6 & 10,3 & 15,2 & 27,1 & $270,8^{*}$ \\
\hline & 2014 & 1,1 & 10,4 & 15,7 & 27,5 & $227,1^{*}$ \\
\hline \multirow[t]{4}{*}{$\mathrm{PCOP}^{\mathrm{b}}$} & 2011 & 2 & 72 & 191,5 & 450 & 3911 \\
\hline & 2012 & 57 & 654,5 & 1312 & 2644,7 & 287020 \\
\hline & 2013 & 26 & 738,7 & 1545 & 2719,7 & 128750 \\
\hline & 2014 & 24 & 483 & 990 & 1983,2 & 45907 \\
\hline \multirow[t]{4}{*}{$\mathrm{TC}^{\mathrm{b}}$} & 2011 & 1 & 12 & 32 & 115 & 1160 \\
\hline & 2012 & 4 & 97 & 224 & 489 & 17871 \\
\hline & 2013 & 2 & 101 & 249 & 503,2 & 13676 \\
\hline & 2014 & 2 & 63 & 176 & 377,7 & 6447 \\
\hline \multirow[t]{4}{*}{$\mathrm{PE}^{\mathrm{a}}$} & 2011 & 0,8 & 12,1 & 17,5 & 22,8 & 53,4 \\
\hline & 2012 & 0,3 & 11,7 & 17,2 & 23,3 & 72,3 \\
\hline & 2013 & 3,4 & 11,5 & 15,1 & 21,0 & 56,6 \\
\hline & 2014 & 2,0 & 10,6 & 13,5 & 18,8 & 50,2 \\
\hline \multirow[t]{4}{*}{$\mathrm{CPCOP}^{\mathrm{a}}$} & 2011 & 0,11 & 1,23 & 2,44 & 4,98 & 528,9 \\
\hline & 2012 & 0,13 & 1,52 & 2,83 & 6,05 & 513,8 \\
\hline & 2013 & 0,04 & 1,49 & 2,74 & 6,57 & 393,4 \\
\hline & 2014 & 0,02 & 0,87 & 1,96 & 3,94 & 1038,1 \\
\hline \multirow[t]{4}{*}{$\mathrm{CEAB}^{\mathrm{a}}$} & 2011 & 42,5 & 100,0 & 100,0 & 100,0 & 100,0 \\
\hline & 2012 & 0,0 & 100,0 & 100,0 & 100,0 & 100,0 \\
\hline & 2013 & 57,8 & 100,0 & 100,0 & 100,0 & 100,0 \\
\hline & 2014 & 14,1 & 100,0 & 100,0 & 100,0 & 100,0 \\
\hline \multirow[t]{4}{*}{$\mathrm{CESB}^{\mathrm{a}}$} & 2011 & 56,6 & 100,0 & 100,0 & 100,0 & 100,0 \\
\hline & 2012 & 61,2 & 100,0 & 100,0 & 100,0 & 100,0 \\
\hline & 2013 & 66,3 & 100,0 & 100,0 & 100,0 & 100,0 \\
\hline & 2014 & 64,4 & 100,0 & 100,0 & 100,0 & 100,0 \\
\hline \multirow[t]{4}{*}{ CESF $^{a}$} & 2011 & 75,2 & 100,0 & 100,0 & 100,0 & 100,0 \\
\hline & 2012 & 73,9 & 100,0 & 100,0 & 100,0 & 100,0 \\
\hline & 2013 & 61,5 & 100,0 & 100,0 & 100,0 & 100,0 \\
\hline & 2014 & 16,2 & 100,0 & 100,0 & 100,0 & 100,0 \\
\hline
\end{tabular}

${ }^{\mathrm{a}}$ Valores percentuais; ${ }^{\mathrm{b}}$ valores absolutos; ${ }^{*}$ valores máximos ultrapassam $100 \%$ porque o número de TC foi maior que o de PCOP.

Fonte: Ministério da Saúde/Sistema de Informação da Atenção Básica.

de 1 apontam para a dificuldade em concluir tratamentos. Quando muito próximo de 1, podem sugerir dificuldade de promover acesso a novos pacientes, e acima de 1 sinalizam que concluíram mais tratamentos do que iniciaram ${ }^{8}$.

A série temporal estudada apresentou significativa variabilidade. Nesse caso, o cálculo do IR evidenciou uma involução nos valores a que poderia estar associada, para além dos problemas de registro, com a reorganização da coleta e a introdução de novos dados no sistema de informações em 2011 sobre a atenção em saúde bucal ${ }^{24}$. Contraditoriamente, estudo com base nos anos 2012 e $2013^{19}$ mostrou que $71,5 \%$ dos usuários da atenção básica no Nordeste estavam satisfeitos com o atendimento ofertado. 
Tabela 2. Valores máximos e mínimos, mediana e distribuição por quartis das variáveis Produto Interno Bruto $(\mathrm{PIB})^{\mathrm{a}}$, Índice de Desenvolvimento Humano Municipal (IDHM) ${ }^{\mathrm{b}}$ e coeficiente de Gini (CG) ${ }^{\mathrm{b}}$ no período de 2011 a 2014 na Paraíba $(\mathrm{n}=124$; municípios/quartil = 31).

\begin{tabular}{lccccrr}
\hline \multicolumn{1}{c}{ Variável } & Ano & Mínimo & Primeiro quartil & Mediana & Terceiro quartil & Máximo \\
\hline & & & $\mathbf{2 5 \%}$ & $\mathbf{5 0 \%}$ & \multicolumn{1}{c}{$\mathbf{7 5 \%}$} & \\
\hline PIB $^{\mathrm{a}}$ & 2011 & 11469,8 & 28751,3 & 48266,2 & 98348,1 & 11639079,7 \\
& 2012 & 12577,6 & 30414,3 & 53156,9 & 110634,0 & 13843818,4 \\
& 2013 & 12881,8 & 34639,4 & 60001,4 & 128159,4 & 14841805,0 \\
IDHM $^{\mathrm{b}}$ & 2010 & 0,51 & 0,57 & 0,59 & 0,61 & 0,76 \\
$\mathrm{CG}^{\mathrm{b}}$ & 2010 & 0,44 & 0,48 & 0,51 & 0,54 & 0,70 \\
\hline
\end{tabular}

${ }^{\mathrm{a}}$ Dado disponível até o ano de 2013, havendo a repetição dos valores desse ano em 2014; ${ }^{\mathrm{b}}$ dados divulgados no Censo $2010 \mathrm{e}$ usados para a análise repetindo o valor para os períodos estudados.

Fonte: IBGE/Censos Demográficos 1991, 2000 e 2010.

Tabela 3. Regressão múltipla binomial negativa do indicador de resolutividade e das variáveis independentes CPCOPa, CEAB e PIB para municípios da Paraíba, entre 2011 e 2014.

\begin{tabular}{lccrc}
\hline Parâmetro & Estimado & $\begin{array}{c}\text { Erro } \\
\text { padrão }\end{array}$ & $\begin{array}{c}\text { RP (IC } \\
\mathbf{9 5 \%})^{\mathbf{b}}\end{array}$ & $\begin{array}{c}\text { Pr }> \\
\text { ChiSq }^{\mathbf{c}}\end{array}$ \\
\hline Intercept & 2,0260 & 0,5380 & & 0,0002 \\
Ano & $-0,1830$ & 0,0632 & 0,83 & 0,0038 \\
& & & $(0,74-0,94)$ & \\
CPCOP & 0,1358 & 0,0590 & 1,14 & 0,0213 \\
& & & $(1,02-1,28)$ & \\
CEAB & 0,0187 & 0,0055 & 1,02 & 0,0008 \\
& & & $(1,01-1,03)$ & \\
PIB & $-0,0000$ & 0,0000 & 0,99 & 0,0037 \\
& & & $(0,99-0,99)$ & \\
\hline
\end{tabular}

${ }^{a}$ CPCOP: cobertura de primeira consulta odontológica programática $=\left(n^{\circ}\right.$ de PCOPs/estimativa da população coberta em junho do respectivo ano) x 100 ; ${ }^{\mathrm{b}}$ razão de prevalência (intervalo de confiança de $95 \%$ ); ${ }^{c}$ p-valor do teste qui-quadrado, considerando nível de significância de 5\%.

Fonte: Dados da pesquisa.

Diante do cálculo do IR proposto neste estudo, sugere-se que o desempenho das equipes de saúde bucal é insuficiente. Conforme demonstrado, a demanda de usuários registrada pelo número de primeiras consultas é imensamente maior do que a capacidade de conclusão dos tratamentos por parte das equipes de saúde. Sendo assim, evidenciou-se que a atenção básica em saúde bucal no estado da Paraíba não conclui efetivamente os tratamentos para os quais deu oportunidade de acesso. Entretanto, não há na literatura nenhum parâmetro que classifique a resolutividade de equipes de saúde segundo o cálculo do IR.

O estudo que propôs o IR relatou a variação deste índice de $68,5 \%$ a $104,6 \%$ em Unidades de Saúde da Família (USF), e de $27,9 \%$ a $71,9 \%$ para as Unidades Básicas de Saúde (UBS) do município de Marília, no estado de São Paulo, região Sudeste do Brasil ${ }^{20}$. Na presente pesquisa, é possível que o modelo de pronto atendimento ${ }^{20}$ tenha influenciado negativamente o valor do IR encontrado. O cálculo do IR apontou desequilíbrio entre o número de primeiras consultas e a quantidade de tratamentos concluídos. Em parte, esse aspecto pode estar relacionado ao processo de trabalho das equipes, que em grande parte priorizam as demandas espontâneas em detrimento das agendadas, o que dificulta a efetivação da longitudinalidade do cuidado, bem como a resolução de urgências na primeira consulta e o abandono do usuário ao plano de tratamento estabelecido. Ainda que o registro de atendimentos de urgências como primeira consulta não seja apropriado, deve-se assumir que algumas equipes de saúde bucal podem fazer uso indevido do indicador de registro de primeiras consultas, o que consequentemente impacta o IR.

Sugere-se que atendimentos eventuais de urgência/emergência são registrados erroneamente como primeira consulta, isto é, sem a devida elaboração de um PPT. Somado a isso, um novo registro de primeira consulta pode ser gerado seis meses após a última visita para o usuário que abandone o tratamento ${ }^{25}$. Ambas as situações provocam elevação do número de primeiras consultas sem que a conclusão do tratamento seja contabilizada, o que impacta significativamente o IR. Além disso, usuários podem abandonar o tratamento após resolverem problemas pontuais identificados na primeira consulta ${ }^{26}$.

Foi verificada a tendência à redução gradativa do percentual de exodontias relacionadas aos demais procedimentos odontológicos básicos, insinuando melhoria da oferta de cuidado e mudança no modelo de atenção em saúde bucal. Essa 
redução mostra que o percentual de exodontias em relação aos procedimentos básicos totais decaiu à medida que as ações de caráter conservador foram ampliadas, associado ao aumento na cobertura de saúde bucal ${ }^{27}$.

As análises efetuadas apontam o IR como um indicador potente para auxiliar a qualificação da atenção básica em saúde bucal, pois identificou falhas nos registros, a exemplo do número excessivo de primeiras consultas e da quantidade de tratamentos concluídos superior ao número de primeiras consultas. Além disso, o IR permitiu mensurar e hierarquizar a produção em termos da capacidade resolutiva, o que pode beneficiar o monitoramento, a avaliação e a definição de metas para o IR.

A literatura sugere que indicadores socioeconômicos interferem na procura da população por serviços de saúde e que possuir garantia mínima de renda pode influenciar positivamente resultados relacionados à saúde bucal ${ }^{28,29}$. A relação entre indicadores socioeconômicos e de saúde bucal ainda não é bem estabelecida, mas tem sido descrita por alguns estudos. Lira Júnior e colaboradores $^{30}$ relatam que o valor do PIB por estados no Brasil tem relação inversa com a cobertura da população na atenção básica. Entretanto, esses autores utilizaram um modelo bivariado, com menor capacidade de explicação do que o modelo estatístico utilizado no presente estudo. A correlação positiva entre IDH e número de primeiras consultas odontológicas programáticas já foi descrita por outro estudo ${ }^{31}$. Além disso, aumento na desigualdade da distribuição de renda pode repercutir negativamente na condição bucal ${ }^{32}$.

A análise estatística buscou identificar fatores associados ao desempenho do IR, considerando dados de produção e variáveis socioeconômicas. Foi observada associação positiva do IR com as coberturas de primeira consulta odontológica programática e de equipes de atenção básica. A relação oferta de serviço versus população interfere nos indicadores de cobertura e de produção. Pimentel e colaboradores ${ }^{33}$ descreveram influ- ência diretamente proporcional da cobertura da população sobre a PCOP. O PIB apresentou relação negativa com o IR, de modo que são necessárias observações adicionais para melhor entendimento dessa relação.

$\mathrm{O}$ indicador de resolutividade se confirma como ferramenta de apoio à gestão em saúde bucal. Entretanto, este estudo apresenta limitações que devem ser consideradas, tais como: qualidade do registro dos dados utilizados (por apresentar falhas de registro/processamento e informações/interpretações distintas dos procedimentos pelos profissionais, gerando inconsistências); delineamento do estudo (que tem como unidade de observação municípios, impossibilitando a identificação dos resultados em cada nível de serviço, fragilizando inferências); impossibilidade de determinar a qualidade dos tratamentos concluídos, a humanização e o acolhimento da prática do cirurgião dentista, bem como sua capacitação e dos profissionais responsáveis por alimentar o banco de dados e a adesão dos usuários ao tratamento, fatores que podem influenciar nos resultados do indicador de resolutividade.

Diante do apresentado, o IR possui potencial para orientar gestores no monitoramento dos serviços ofertados e permitir a criação de novos mecanismos de avaliação e controle, de modo a reduzir deficiências no processo de trabalho na atenção básica em saúde bucal. Novos estudos devem ser desenvolvidos para a incluir dados de anos mais recentes disponibilizados na nova plataforma do Sistema de Informações do Departamento de Atenção Básica (e-SUS AB).

\section{Conclusões}

A resolutividade em saúde bucal na atenção básica foi influenciada positivamente por fatores relacionados à cobertura. $\mathrm{O}$ cálculo do IR neste estudo demonstrou que a saúde bucal na atenção básica não concluiu efetivamente os tratamentos para os quais de oportunidade de acesso. 


\section{Colaboradores}

CPL Santiago trabalhou na concepção, no delineamento da pesquisa e na redação do artigo; GMB Ambrosano trabalhou na análise e interpretação dos dados, na revisão crítica do artigo e aprovação da versão final; DFB Cavalcante participou da revisão crítica do artigo e da aprovação da versão final; AC Pereira contribuiu na concepção, no delineamento da pesquisa, na revisão crítica do artigo e aprovação da versão final; EHG Lucena trabalhou no delineamento da pesquisa, na aquisição dos dados, na revisão crítica do artigo e aprovação da versão final; YW Cavalcanti participou do delineamento da pesquisa, da interpretação dos dados, da revisão crítica do artigo e aprovação da versão final; WWN Padilha colaborou na concepção, no delineamento da pesquisa, na revisão crítica do artigo e aprovação da versão final.

\section{Agradecimentos}

À Coordenação de Aperfeiçoamento de Pessoal de Nível Superior (CAPES).

\section{Referências}

1. Brasil. Conselho Nacional de Secretários de Saúde (CONASS). Atenção Primária e Promoção de Saúde. [documento da Internet]; 2011 [acessado $2017 \mathrm{dez}$ 05]. Disponível em: http://www.conass.org.br/bibliotecav3/pdfs/colecao2011/livro_3.pdf

2. Pucca Junior G, Gabriel M, Araujo M, Almeida F. Ten years of a national oral health policy in Brazil: innovation, boldness, and numerous challenges. J Dent Res 2015; 94(10):1333-1337.

3. Arantes LJ, Shimizu HE. Evaluation of the implementation of the Primary Health Care Master Plan in Unaí, Brazil. J Public Health 2012; 20(3):227-233.

4. Oliveira AEF, Reis RS. Gestão pública em saúde: monitoramento e avaliação no planejamento do SUS 2016. [acessado 2017 Dez 05]. Disponível em: http://www. unasus.ufma.br/site/files/livros_isbn/isbn_gp11.pdf.

5. Patera N, Wild C. Linking public health research with policy and practice in three European countries. $\mathrm{J} \mathrm{Pu}$ blic Health 2015; 21(5):473-479.

6. Cruz DF, Prado RL, Valença AMG, Santos ML. A linha do cuidado em saúde bucal no município de João Pessoa: uma análise de indicadores. Pesqui Bras Odontopediatria Clin Integr 2011; 11(2):291-295.

7. Madeira LM. Avaliação de políticas públicas 2014. [acessado 2017 Dez 05]. Porto Alegre: Universidade Federal do Rio Grande do Sul. Disponível em: https:// www.ufrgs.br/cegov/files/pub_37.pdf

8. Brasil. Ministério da Saúde (MS). Manual instrutivo PMAQ - para as Equipes de Atenção Básica (Saúde da Família, Saúde Bucal e Equipes Parametrizadas) e Nasf. [acessado 2019 Abr 17]. Disponível em: http:// bvsms.saude.gov.br/bvs/publicacoes/manual_instrutivo_pmaq_atencao_basica.pdf

9. Turrini NRT, Lebrão ML, Cesar CLG. Resolutividade dos serviços de saúde por inquérito domiciliar: percepção do usuário. Cad Saude Publica 2008; 24(3):919.

10. Costa JP, Jorge MSB, Vasconcelos MGF, Paula ML, Bezerra IC. Resolubilidade do cuidado na atenção primária: articulação multiprofissional e rede de serviços. Saúde Debate 2014; 38(103):733-743.

11. Kusma SZ, Moysés ST, Moysés SJ. Health promotion: perspectives for evaluation of oral health in primary healthcare. Cad Saude Publica 2012; 28:9-19.

12. Secretaria de Políticas de Saúde. Departamento de Atenção Básica. Programa Saúde da Família. Rev Saude Publica 2000; 34(3):316-319.

13. van der Graaf P, Forrest LF, Adams J, Shucksmith J, White M. How do public health professionals view and engage with research? A qualitative interview study and stakeholder workshop engaging public health professionals and researchers. BMC Public Health 2015; 17(1):892.

14. Bogaert P, Van Oyen H. An integrated and sustainable EU health information system: national public health institutes' needs and possible benefits. Arch Public Health 2017; 75(1):3.

15. Franco JLF. Indicadores demográficos e de saúde: a importância dos sistemas de informação. UNA-SUS/ UNIFESP 2010. [acessado 2017 Dez 05]. Disponível em: http://www.unasus.unifesp.br/biblioteca_virtual/ esf/1/modulo_politico_gestor/Unidade_8.pdf 
16. Organização Pan-Americana da Saúde (OPAS). Indicadores de saúde. Elementos conceituais e práticos. Washington; 2018. [acessado 2021 Jun 23]. Disponível em: https://iris.paho.org/bitstream/handle/10665.2/49057/ 9789275720059_por.pdf?sequence $=5$

17. Brasil. Ministério da Saúde (MS). Portal e-Gestor AB. Relatório histórico de cobertura. [acessado 2021 Jun 23]. Disponível em: https://egestorab.saude.gov.br/ paginas/acessoPublico/relatorios/relHistoricoCobertura.xhtml

18. Cruz MM. Avaliação de políticas e programas de saúde: contribuições para o debate. In: Mattos RA, Baptista TWF. Caminhos para análise das politicas de saúde. Porto Alegre; 2011.p. 181-199.

19. Protasio APL, Gomes LB, Machado LS, Valença AMG. Satisfação do usuário da Atenção Básica em Saúde por regiões do Brasil: $1^{\circ}$ ciclo de avaliação externa do PMAQ-AB. Cien Saude Colet 2017; 22(6):1829-1844.

20. Bulgareli J, Cortellazzi KL, Ambrosano GMB, Meneghim MC, Faria ET, Mialhe FL, Pereira AC. Resolubility in oral health for primary care as an instrument for the evaluation of health systems. Cien Saude Colet 2014; 19(2):383-391.

21. Instituto Brasileiro de Geografia e Estatística (IBGE). Estimativas de população. [acessado 2017 Dez 05]. Disponível em: https://sidra.ibge.gov.br/pesquisa/estimapop/tabelas

22. E-gestor atenção básica. Informação e gestão da Atenção Básica 2017. [acessado 2017 Dez 05]. Disponível em: https://egestorab.saude.gov.br/paginas/acessoPublico/relatorios/relHistoricoCoberturaAB.xhtml

23. Brasil. Ministério da Saúde (MS). Critérios e parâmetros para o planejamento e programação de ações e serviços de saúde no âmbito do Sistema Único de Saúde - Parâmetros SUS 2015. [acessado 2017 Dez 05]. Disponível em: http://www.fehosp.com.br/app/webroot/ files/manuais/5ffa8d1e03f7edb01e1eed7b07178cfb. pdf

24. Brasil. Ministério da Saúde (MS). Secretaria de Atenção à Saúde. Departamento de Atenção Básica. Coordenação-Geral de Acompanhamento e Avaliação. Instrutivo do Sistema de Informação da Atenção Básica - SIAB. 2a versão 2011. [acessado 2017 Dez 05]. Disponível em: http://189.28.128.100/dab/docs/geral/ Instrutivo_SIAB_2011_V2.pdf

25. Brasil. Ministério da Saúde (MS). E-SUS: manual do sistema com coleta de dados simplificada-CDS (versão 2.0). 2015. [acessado 2017 Dez 05]. Disponível em: http://189.28.128.100/dab/docs/portaldab/documentos/Manual_CDS_2_0_versao_preliminar_ago_2015. pdf

26. Oliveira MM, Andrade SSCA, Campos MO, Malta DC. Fatores associados à procura de serviços de saúde entre escolares brasileiros: uma análise da Pesquisa Nacional de Saúde do Escolar (PeNSE), 2012. Cad Saude Publica 2015; 31(8):1603-1614.

27. Oliveira PMC, Menezes LMB, Lima Santrain MV, Almeida PC, Almeida MEL. Indicadores de saúde bucal da atenção básica no estado do Ceará: análise crítica. Cadernos ESP, Ceará 2011; 5(1):29-36.
28. Chen YA, Quiñonez C. Basic income guarantee: a review of implications for oral health. J Public Health Dent 2017; 78(1):56-62.

29. Madruga CRM, Soares RSC, Cardoso AMR, Góes PSA, Cavalcanti AL. Access to oral health services in areas covered by the family health strategy, Paraíba, Brazil. Pesqui Bras Odontopediatria Clin Integr 2017; 17(1):1-10.

30. Lira Júnior R, Cavalcanti YW, Brito DBA, Lima AA, Padilha WWN. Indicadores socioeconômicos e desigualdades em Saúde Bucal no Brasil. Rev Odontol Bras Central 2011; 20(52):20-24.

31. Pimentel BV, Zermiani TC, Ditterich RG, Pecharki GD. A utilização dos indicadores de saúde bucal e de desenvolvimento humano no monitoramento da atenção básica nos municípios da região metropolitana de Curitiba-PR. Revista de Saúde Pública do Paraná 2014; 15(3):42-52.

32. Goulart MA, Vettore MV. Is the relative increase in in come inequality related to tooth loss in middle-aged adults? J Public Health Dent 2016; 76(1):65-75.

33. Pimentel FC, Albuquerque PC, Martelli PJL, Acioli RML, Souza WV. Analysis of oral health indicators of Pernambuco: performance of cities according to size population, population enrolled in the Information System for Primary Care and proportion in the Family Health Strategy. Cad Saude Colet 2014; 22(1):5461.

Artigo apresentado em 26/11/2018

Aprovado em 18/11/2019

Versão final apresentada em 20/11/2019

Editores-chefes: Romeu Gomes, Antônio Augusto Moura da Silva 
